\title{
Applying Capacitive Energy Storage for In Situ Manipulation of Magnetization in Ordered Mesoporous Perovskite-Type LSMO Thin Films
}

Christian Reitz, ${ }^{,,+, \ddagger}$ Di Wang, ${ }^{\dagger, \ddagger}$ Daniela Stoeckel, ${ }^{\S, \perp}$ Andre Beck, ${ }^{\#}$ Thomas Leichtweiss, ${ }^{\perp}$ Horst Hahn, ${ }^{\dagger, \S}$ and Torsten Brezesinski ${ }^{*, \dagger}$

${ }^{\dagger}$ Institute of Nanotechnology, ${ }^{\ddagger}$ Karlsruhe Nano Micro Facility, and ${ }^{\#}$ Institute of Solid State Physics, Karlsruhe Institute of Technology, Hermann-von-Helmholtz-Platz 1, 76344 Eggenstein-Leopoldshafen, Germany

${ }^{\S}$ Department of Chemistry, University of Marburg, Hans-Meerwein-Straße, 35032 Marburg, Germany

Institute of Physical Chemistry, Justus-Liebig-University Giessen, Heinrich-BuffRing 17, 35392 Giessen, Germany

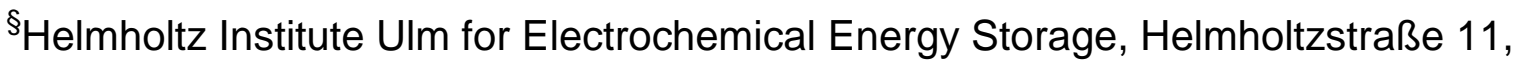
89081 Ulm, Germany

\section{Keywords}

Block copolymer templating, mesoporosity, lanthanum strontium manganese oxide, perovskite, electrolyte gating, double-layer charging, tuning of magnetism 


\begin{abstract}
Mesostructured non-silicate materials, particularly mixed-metal oxides, are receiving much attention in recent years due to their potential for numerous applications. Via polymer-templating method, perovskite-type lanthanum strontium manganese oxide $\left(\mathrm{La}_{1-x} \mathrm{Sr}_{x} \mathrm{MnO}_{3}\right.$, LSMO, with $x \approx 0.15$ to 0.30 ) with a continuous 3D cubic network of $23 \mathrm{~nm}$ pores is prepared in thin film form for the first time. Characterization results from grazing incidence $X$-ray scattering, $X$-ray photoelectron spectroscopy, Rutherford backscattering spectrometry, and electron microscopy and tomography show that the dip-coated sol-gel-derived films are of high quality in terms of composition and morphology, and that they are stable to over $700^{\circ} \mathrm{C}$. Magnetic and magnetotransport measurements demonstrate that the material with the highest strontium concentration is ferromagnetic at room temperature and exhibits metallic resistivity behavior below $270 \mathrm{~K}$. Besides, it behaves differently from epitaxial layers (e.g., enhanced low-field magnetoresistance effect). It is also shown that carriers (electrons and holes) can be induced into the polymer-templated mesostructured LSMO films via capacitive double-layer charging. This kind of electrostatic doping utilizing ionic liquid gating causes large relative changes in magnetic susceptibility at room temperature and is a viable technique to tune the magnetic phase diagram in situ.
\end{abstract}




\section{Introduction}

Mixed-valence manganese oxides with a perovskite-type structure constitute an important class of materials with potential for applications in solid-oxide fuel cells, magnetic field sensors, information storage, and so forth. ${ }^{1-5}$ Here we focus on lanthanum strontium manganese oxide ( $\left.\mathrm{La}_{1-x} \mathrm{Sr}_{x} \mathrm{MnO}_{3}, \mathrm{LSMO}\right)$, which is the most prevalent and best-studied mixed-valence manganese oxide. LSMO can be described as a solid solution between $\mathrm{La}^{3+} \mathrm{Mn}^{3+} \mathrm{O}_{3}$ and $\mathrm{Sr}^{2+} \mathrm{Mn}^{4+} \mathrm{O}_{3}$ and is a strong electron-correlated colossal magnetoresistive (CMR) material. ${ }^{6-9}$ Because of the correlation between magnetic interactions and electronic transport properties, the carrier density, and therefore the magnetization, can be tuned via electrostatic doping utilizing either solid or liquid electrolyte gating. ${ }^{10-16}$ It should be noted, nevertheless, that depending upon the material class, in situ manipulation of magnetization can also be achieved via interfacial strain mediation or lithium insertion reaction. ${ }^{17-25}$ For applications, tuning of physical properties is important, and these approaches may lead to the development of new device concepts (e.g., for next-generation logic and memory). Assuming that the change in carrier density in LSMO determines the magnitude of the magnetization modulation, the specific surface area should play a significant role.

In the present work, we show, to our knowledge for the first time, that large-pore mesoporous LSMO can be prepared as a thin film by using an evaporation-induced self-assembly (EISA) process. ${ }^{26-28}$ In the past two decades, this synthetic method has proven particularly efficient for amorphous silica-based compounds and binary metal oxides. ${ }^{29-37}$ However, the preparation of either cubic or hexagonal mixed-metal oxides with highly crystalline walls is known to be challenging in general for a number of reasons, such as the lack of control over both the hydrolysis and condensation rates and the crystallization.

Here we make use of the excellent templating properties of an amphiphilic poly (ethylene oxide)-based block copolymer to produce a series of thermally stable perovskite-type LSMO thin films with an open cubic pore network. ${ }^{38-40}$ Their structural and compositional uniformity makes them good "model" materials to study (i) the effect that the unique combination of nanocrystallinity and mesoporosity has on the magnetotransport properties and (ii) whether a high surface-to-volume ratio represents an advantage in the manipulation of magnetization upon electrochemical charge storage via double-layer capacitance. Overall, we show that the polymertemplated mesostructured LSMO thin films exhibit enhanced low-field magnetoresistance effect (compared with bulk LSMO) and that electrostatic hole and electron doping by ionic liquid gating allows large tuning of the total magnetization and Curie temperature. 


\section{Experimental Section}

\section{LSMO Thin Film Synthesis}

In a typical synthesis (e.g., LSMO30), $\mathrm{H}\left[\mathrm{C}\left(\mathrm{CH}_{3}\right)_{2} \mathrm{CH}_{2}\right]_{107} \mathrm{C}_{6} \mathrm{H}_{4}\left(\mathrm{OCH}_{2} \mathrm{CH}_{2}\right)_{150} \mathrm{OH}(50$ $\mathrm{mg}$ ) dissolved in a mixture of ethanol $(1.5 \mathrm{~mL}), 2$-methoxyethanol $(0.5 \mathrm{~mL})$, tetrahydrofuran $(0.15 \mathrm{~mL})$, glacial acetic acid $(0.1 \mathrm{~mL})$, and deionized water $(0.15$ $\mathrm{mL}$ ) was combined with $\mathrm{Mn}(\mathrm{OAc})_{2} \times 4 \mathrm{H}_{2} \mathrm{O}(119 \mathrm{mg}), \mathrm{La}\left(\mathrm{NO}_{3}\right)_{3} \times 6 \mathrm{H}_{2} \mathrm{O}(147 \mathrm{mg})$, and $\mathrm{Sr}\left(\mathrm{NO}_{3}\right)_{2}(31 \mathrm{mg})$. After stirring the clear solution for $30 \mathrm{~min}$ at room temperature, thin films were produced by dip-coating on $\mathrm{Si}(001)$ and quartz glass substrates at relative humidities ranging from 30 to $35 \%$. Then they were transferred to an oven at $140^{\circ} \mathrm{C}$ for $15 \mathrm{~min}$, followed by heating to $300^{\circ} \mathrm{C}$ over a period of $90 \mathrm{~min}$. After $12 \mathrm{~h}$ ageing at $300^{\circ} \mathrm{C}$, the films were subjected to calcination at $700^{\circ} \mathrm{C}$ for 2 min to fully combust the organics and to achieve crystallization of the inorganic walls.

\section{Characterization Methods}

Scanning electron microscopy (SEM) was performed on a MERLIN from Carl Zeiss operated at $5 \mathrm{keV}$. Transmission electron microscopy (TEM) was performed on an aberration corrected FEI Titan ${ }^{3}$ 80-300 microscope. The microscope was operated at $300 \mathrm{keV}$ in TEM mode for high-resolution TEM (HRTEM) and in nanoprobe mode for scanning TEM (STEM). The powder samples were dispersed on copper grids covered by an amorphous carbon film. For electron tomography, colloidal Au particles around $15 \mathrm{~nm}$ in size, serving as fiducial markers, were deposited, followed by acquiring series high-angle annular dark-field (HAADF) STEM images with single axis tilting the sample from $-76^{\circ}$ to $+76^{\circ}\left(1.5^{\circ}\right.$ tilting steps). The tilt series images were first aligned in IMOD by tracking 20 fiducial markers until the final mean residual error was 0.36 pixel. ${ }^{41}$ The 3D structure was subsequently reconstructed from the aligned tilt series using the SIRT algorithm in Inspect 3D. Reconstructed volume was segmented in solid phase and void space using AMIRA software (Visage Imaging $\mathrm{GmbH}$ ). The segmented image stack was subjected to Gaussian smoothing and submitted to a restoration process during which isolated elements of solid in the void space and of void space in the solid phase were corrected. 3D rendering of the binarized data set was also realized by use of AMIRA. The surface area of designated segments was analyzed by means of the "SurfaceArea" function (on the basis of the rendered triangular approximation of interfaces between the segmented sections). To evaluate the reconstructed pore space/wall phase statistically, the chord length distributions were calculated using an in-house software written with Visual Studio C\# 2008 (Microsoft Corporation). Random points were generated in the segmented image stacks and the linear skeleton-to-skeleton (or pore-to-pore) distance was determined for 16 equispaced directions, as described in refs 42,43 . The reconstructed sample was skeletonized based on an iterative thinning algorithm, available as ImageJ plug-ins (Skeletonize3D and AnalyzeSkeleton). Skeletonization reduces the pore space to a medial axis while retaining topological and geometrical information. The resulting topological skeleton 
was evaluated by determining the total number of junctions $\left(n_{\mathrm{j}}\right)$, where $n_{\mathrm{t}}$ is the number of triple-point junctions, $n_{\mathrm{q}}$ is the number of quadruple-point junctions, and $n_{\mathrm{x}}$ represents the number of higher-node junctions. The average pore coordination number ( $Z_{\mathrm{av}}$, average number of pores attached to a junction) is:

$$
Z_{\mathrm{av}}=3 \frac{n_{\mathrm{t}}}{n_{\mathrm{j}}}+4 \frac{n_{\mathrm{q}}}{n_{\mathrm{j}}}+5 \frac{n_{\mathrm{x}}}{n_{\mathrm{j}}}
$$

In addition, the pore-level tortuosity was evaluated by the analysis of individual branches of the derived skeleton. The tortuosity is defined as the ratio of the geodesic distance of a pore $\left(d_{i}\right)$ divided by the Euclidean distance between the pore entrance and exit $\left(d_{\text {eucl, }}\right)$. From this, the average tortuosity can be calculated according to:

$$
\tau=\frac{1}{\mathrm{j}} \sum_{\mathrm{i}=1}^{\mathrm{j}} \frac{d_{\mathrm{i}}}{d_{\text {eucl, } \mathrm{i}}}
$$

Grazing incidence small-angle X-ray scattering (GISAXS) was performed on beamline P03 at HASYLAB at DESY $\left(22.6 \times 23.3 \mu \mathrm{m}^{2}\right.$ beam size, $\lambda=0.1088 \mathrm{~nm}$, $\left.d_{\text {sample/detector }}=3776 \mathrm{~mm}\right)$ by use of a DECTRIS Pilatus $1 \mathrm{M}$ area detector $(981 \times 1043$ pixels, $172 \times 172 \mu \mathrm{m}^{2}$ pixel size). The angle of incidence was set to $0.2^{\circ}$. For data analysis, DPDAK 1.2.0 software was used. ${ }^{44}$ Grazing incidence wide-angle X-ray scattering (GIWAXS) was performed on a Bruker D8 Discover diffractometer equipped with a Soller slit and LynxEye strip detector $\left(\lambda=0.15418 \mathrm{~nm}, d_{\text {sample/detector }}\right.$ $=26 \mathrm{~cm}$ ). For Rietveld refinement, FullProf software was used. The reflections were described by the Thompson-Cox-Hastings pseudo-Voigt profile function using a polynomial background model. X-ray photoelectron spectroscopy (XPS) was performed on a VersaProbe PHI 5000 Scanning ESCA Microprobe from Physical Electronics with an Al- $\mathrm{K}_{\alpha}$ radiation source and hemispherical electron energy analyzer. The $\mathrm{C} 1 \mathrm{~s}$ signal from adventitious hydrocarbon at $284.8 \mathrm{eV}$ was used as the energy reference to correct for charging. Except for plasmon loss and Auger peaks, the full width at half maximum (fwhm) was constrained to be equal for all peaks of a given core level during fitting, and all spectra were baseline corrected using the Shirley method. Rutherford backscattering spectrometry (RBS) was performed with $2 \mathrm{MeV}^{4} \mathrm{He}^{+}$ions, with the parallel beam (at normal incidence) irradiating a cross-sectional area of $1 \mathrm{~mm}^{2}$. The backscattered ions were analyzed at an angle of $152^{\circ}$. The ion beam divergence was smaller than $0.02^{\circ}$. The solid angle of the detector was in the range of $10^{-3} \mathrm{sr}$. For data analysis, SIMNRA software was used. ${ }^{45}$ Magnetic susceptibility measurements were performed on a Quantum Design MPMS 3 superconducting quantum interference device (SQUID) magnetometer. The magnetotransport properties were analyzed by means of a Quantum Design MPMS XL-5 SQUID magnetometer. For van der Pauw four-probe measurements, the LSMO thin films on quartz glass substrate were cleaved into smaller pieces to fit the dimension of the sample tube. Liquid electrolyte gating experiments were performed inside a SQUID magnetometer by use of a custom-built 
sample tube. ${ }^{10,17}$ Two-electrode cells with a high-surface-area carbon fiber cloth counter-electrode were assembled inside an Ar-filled glovebox with both $\left[\mathrm{O}_{2}\right]$ and $\left[\mathrm{H}_{2} \mathrm{O}\right]<1 \mathrm{ppm}$. The (non-aqueous) electrolyte was diethylmethyl(2-

methoxyethyl)ammonium bis(trifluoromethylsulfonyl)imide, and glass microfiber filter paper (Whatman, GF/A) served as the separator. Cyclic voltammetry was performed by use of an Autolab PGSTAT302 potentiostat.

\section{Results and Discussion}

The LSMO thin films employed here were prepared by block copolymer templating using common inorganic salt precursors. After their deposition by dip-coating onto silicon and glass substrates, they were slowly heated in air to $700{ }^{\circ} \mathrm{C}$ to combust all of the organic constituents (to make the material porous) and to achieve single phase LSMO while retaining nanoscale structure. Although a series of thin films with varying strontium contents from $x \approx 0.15$ to 0.30 (denoted as LSMO15, LSMO225, and LSMO30) were fabricated during the course of this work, we specifically focus on the material with the highest concentration (i.e., LSMO30) in the following. Nevertheless, the characterization results for LSMO15 and LSMO225 from SEM, GIWAXS, XPS, and RBS, can be found in the Supporting Information (see Fig. S1S4). These data emphasize their structural and chemical homogeneity at the nanometer and micrometer length scales.

The 3D structural characterization was performed by a combination of electron microscopy, including SEM, TEM, and STEM tomography, and GISAXS. Fig. 1 shows results for LSMO30 from TEM and SEM studies. Cross-sectional SEM reveals a well-developed cubic mesophase without major defects and further establishes that the pore network persists throughout the bulk. The pores have an oblate spheroidal shape with an aspect ratio $(z / x)$ of about 0.3 due to unidirectional film contraction mainly during thermal annealing (see also section on GISAXS below). According to STEM and TEM, the average lateral pore size is $23 \mathrm{~nm}$. STEM also confirms the homogeneity of composition, and HRTEM and selected area electron diffraction (SAED) demonstrate both the polycrystalline nature and the phase purity of the sol-gel-derived material (see also bright-field TEM and STEM images at different magnifications in Fig. S5). 


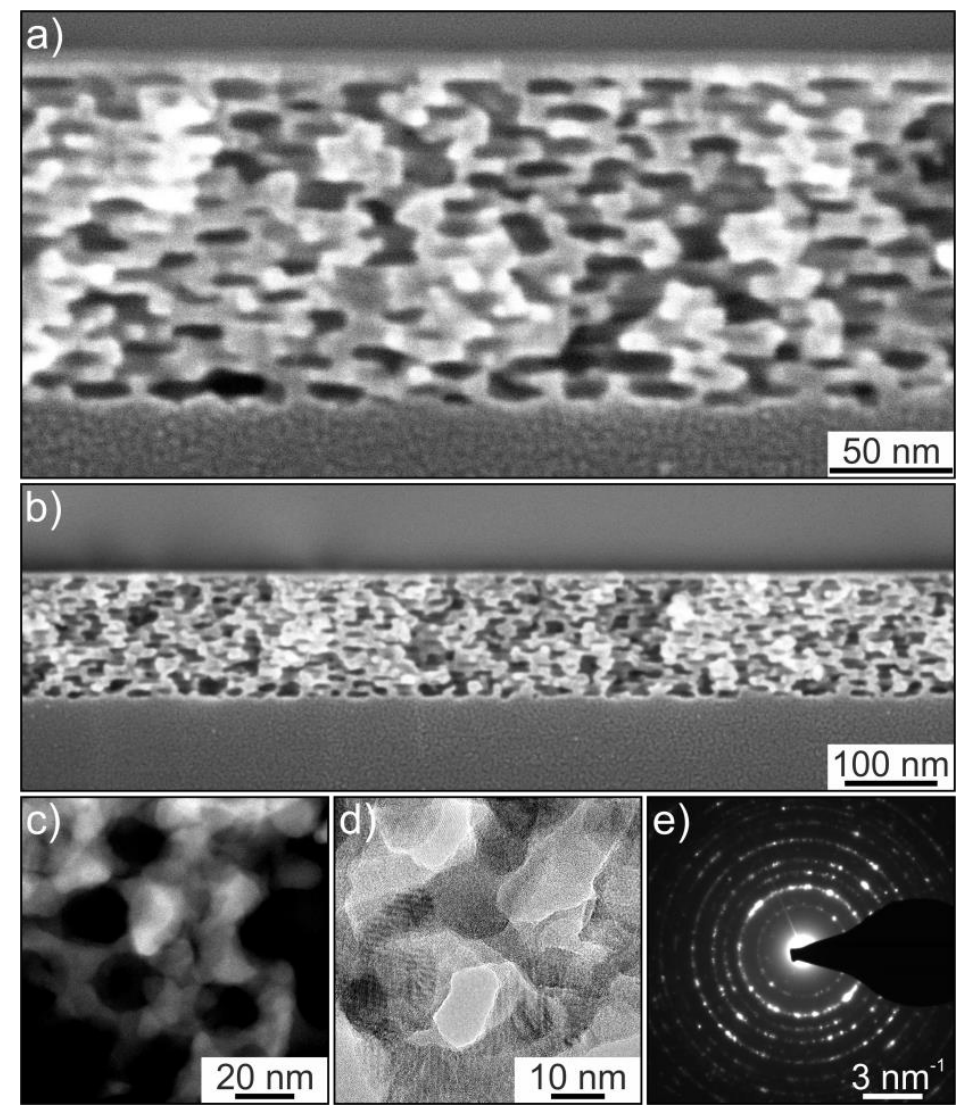

Figure 1. Electron microscopy of polymer-templated mesostructured LSMO30 thin films. (a, b) Cross-sectional SEM images at different magnifications and (c) HAADFSTEM image showing oblate pores arranged in a cubic array. (d) HRTEM image and (e) SAED pattern demonstrating the nanocrystallinity and phase purity of material after heating at $700^{\circ} \mathrm{C}$.

Fig. 2 presents a STEM tomography reconstruction of the 3D structure. At first glance - and taking into account the microscopy data shown in Fig. 1 - the pore/solid architecture seems to be homogeneous. However, to quantify the degree of heterogeneity, a statistical approach is required. Thus, a chord length distribution (CLD) analysis was performed and combined with a skeletonization approach. For CLDs, the pore wall interfaces are typically scanned with chords of different length and the relative frequency with which a defined chord (distance between opposing walls) appears in the pore space is displayed. It can be used to describe the void volume without the necessity of predefining a specific geometry. For this reason, CLD analyses are very useful, particularly for characterizing disordered porous materials, where the assignment of a pore model is either not possible or can falsify the results. Overall, CLDs give the prevailing length scales within the material, but they cannot be directly correlated with an average model-based pore size. As is evident from Fig. 2, the polymer-templated mesostructured LSMO30 thin films exhibit a relatively narrow (monomodal) CLD for the walls, while that of the pore space is broader, with a shoulder around $30 \mathrm{~nm}$. The mode of the CLDs lies at 12.5 
and $17.5 \mathrm{~nm}$ for solid (walls) and void (pores), respectively. The broadening of the pore CLD is caused by a small fraction of "long" chords within the pore space. These might stem from cavities with larger dimensions, but seem rather due to two or more pores that are connected along a line (see also cross-sectional SEM images in Fig. 1). If the "long" chords were indicating the presence of voluminous pores, they should be visible in the 2D slices through the reconstructed structure. However, the slices shown in Fig. 2 do not reveal any significant heterogeneity, but instead confirm the existence of interconnected elongated pores.

Both the pore (inter)connectivity and tortuosity were analyzed in more detail as well. For disordered materials, such parameters are usually derived by fitting bulk data to a model. Our approach - using tomographic methods to reconstruct the pore space and analyzing the connectivity via skeletonization analysis - gives much more realistic results. The average pore coordination number $(Z)$ obtained is 3.28 , with a vast majority of pores (77\%) having a coordination number of $n=3(n=4,18 \% ; n \geq$ $5,5 \%)$. These values are even slightly higher than those usually found for siliceous materials used for chromatographic applications. ${ }^{46-48}$ Consequently, it is reasonable to assume that the pore network of the LSMO30 thin films allows for facile mass transport through the material.

Finally, the volume-specific surface area was determined from the rendered isosurface of two different cuboids cut from the reconstructed (non-symmetrical) 3D volume. Surface areas ranging from 191 to $201 \mathrm{~m}^{2} / \mathrm{cm}^{3}$ were obtained when using a generation method without surface smoothing. In contrast, the surface area was lower by approx. $9 \%$ when applying a constrained smoothing algorithm. However, given that the segmentation process already involved some interface smoothing, the calculated values rather underestimate the actual volume-specific surface area of the material. It should be noted, nevertheless, that they were determined from very small volumes $\left(\approx 2.1 \times 10^{-15} \mathrm{~cm}^{3}\right)$, and therefore should be treated as estimates only; yet, they are consistent with those found for other large-pore mesoporous metal oxide thin films prepared using the same polymer structure-directing agent. ${ }^{49,50}$ 

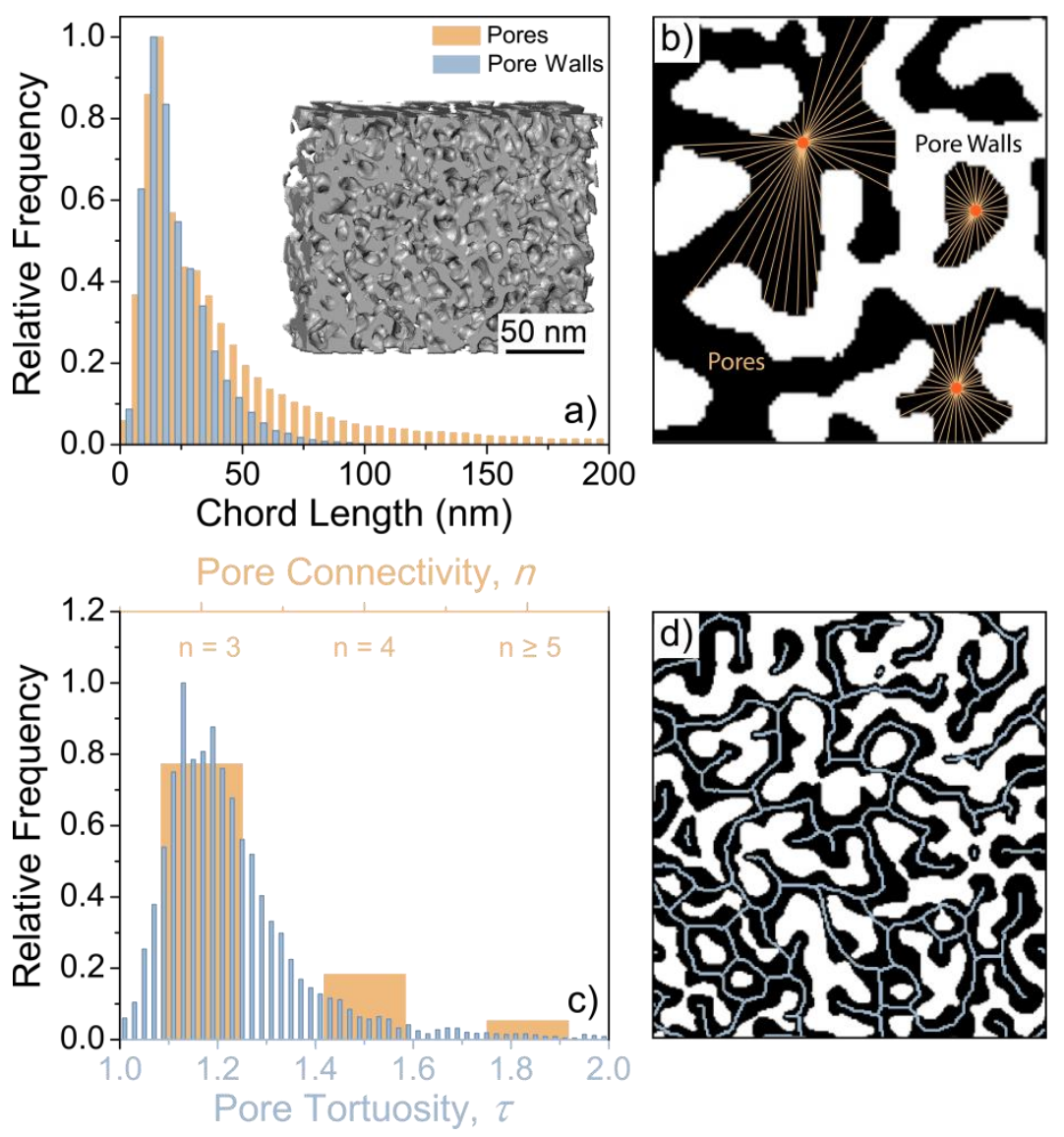

Figure 2. Morphological characterization of polymer-templated mesostructured LSMO30 thin films. (a) CLDs of pore space and solid phase. A reconstructed part of the material (from STEM tomography) with dimensions of $155 \times 193 \times 69 \mathrm{~nm}^{3}$ is shown in the inset. (b) Chords scanning the pore wall interface are spread from random points (orange dots) within the pore space (black) or solid phase (white). (c) Results from topological analysis of the pore space using the skeletonization approach. (d) 2D slice through the reconstructed pore space. The derived pore skeleton is shown in pale blue.

Fig. 3 shows GISAXS data obtained on polymer-templated mesostructured LSMO30 thin films before and after crystallization. As is seen, the amorphous material produces a pattern with strong scattering maxima both in the $\mathbf{q}_{z}$ and $\mathbf{q}_{y}$ direction. However, because of the relatively weak preferred orientation to the substrate, it cannot be clearly assigned to a specific mesophase. Nevertheless, GISAXS confirms the structural homogeneity and high degree of pore ordering, the latter of which is notable, particularly for this kind of self-assembled material. We note that mixedmetal oxides prepared by soft-templating method usually tend to have an ill-defined porosity. Part of the reason for this is the lack of control over the hydrolysis and condensation reactions of the inorganic precursors and the difficulty of crystallizing the nanometer-thick walls while retaining the original pore structure of the material. The pattern shape further indicates a lattice (or volume) contraction of more than 
$70 \%$ by $500{ }^{\circ} \mathrm{C}$. This result is in agreement with the presence of oblate pores with an aspect ratio of about 0.3 . After crystallization only the scattering along $\mathbf{q}_{\mathrm{y}}$ is preserved, which is due to the morphological anisotropy of the thin films. However, as already indicated by cross-sectional SEM, the lateral (in plane) pore ordering is little affected; the maxima are still distinct without apparent broadening or shift in position (see horizontal line cuts in Fig. 3). Thus, we conclude that the different LSMO thin films are well-defined from a structural point of view and can be considered good "model" materials.
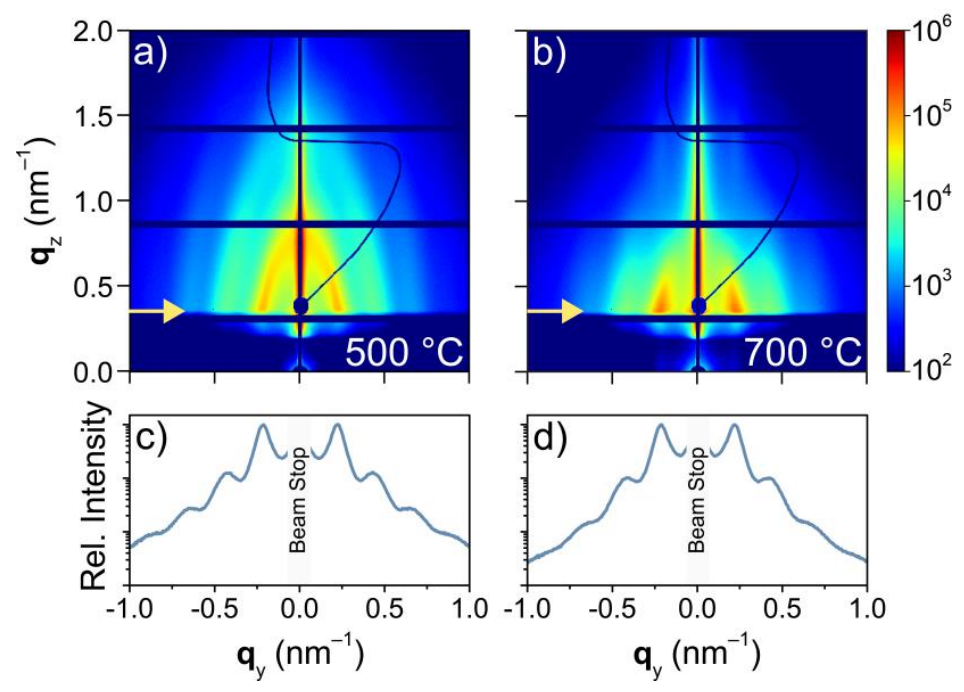

Figure 3. Ex situ GISAXS patterns of polymer-templated mesostructured LSMO30 thin films (a) before and (b) after crystallization of the inorganic walls. (c, d) Horizontal line cuts along $\mathbf{q}_{\mathbf{y}}$ (yellow arrows) indicating that the lateral film structure remains virtually unaffected.

The crystallinity, chemical composition, and bonding configuration of the polymertemplated mesostructured LSMO thin films prepared in this work were investigated by a combination of GIWAXS, XPS, and RBS. The data obtained for LSMO30 as well as for LSMO15 and LSMO225 are shown in Fig. 4 and Fig. S2-S4, respectively. Fig. 4a presents a GIWAXS pattern after heating at $700^{\circ} \mathrm{C}$. Structural refinement confirmed the films to be of Pnma type (isomorphous to $\mathrm{GdFeO}_{3}$ ). A schematic of the orthorhombic unit-cell is shown in the inset of Fig. 4a. The quality of refinement was evaluated by the magnitude of both the weighted profile $R$-factor $\left(R_{\mathrm{wp}}=10.50 \%\right)$ and the goodness-of-fit parameter $\left(x^{2}=0.89\right)$. The fact that the discrepancy values are relatively low indicates that the fit is good and further corroborates the phase purity of the sol-gel-derived material. The average crystallite size from line broadening was determined to be $(14 \pm 2) \mathrm{nm}$, regardless of composition. This also implies that there is no mismatch between the average crystallite size and the wall thickness, thereby helping to explain why the pore/solid architecture is well preserved after crystallization. 
Both the chemical composition and bonding were analyzed in more detail by XPS. Detailed spectra of the La 3d, Mn 2p, and Sr 3d regions are shown in Fig. 4b-d, along with the peak fits; survey spectra are presented in Fig. S3. The La 3d spectrum exhibits a complex satellite structure because of hybridization between the $\mathrm{O} 2 \mathrm{p}$ valence band and the formally empty $4 \mathrm{f}$ states of $\mathrm{La}^{3+}$. It was deconvoluted into 8 peaks. The strongest components, with an area ratio of $3: 2$ for the $3 d_{5 / 2}$ and $3 d_{3 / 2}$ peaks, are located at $(833.6 \pm 0.1) \mathrm{eV}$ and $(850.6 \pm 0.1) \mathrm{eV}$. The peaks at $(835.7 \pm$ $0.1) \mathrm{eV}$ and $(852.3 \pm 0.1) \mathrm{eV}$ as well as at $(838.0 \pm 0.1) \mathrm{eV}$ and $(854.8 \pm 0.1) \mathrm{eV}$ correspond to the bonding and antibonding components of the final state with charge transfer. Besides, there are also 2 relatively broad peaks centered at $(847.6 \pm 0.1)$ $\mathrm{eV}$ and $(851.1 \pm 0.1) \mathrm{eV}$ due to a La $3 \mathrm{~d}_{5 / 2}$ plasmon loss and a Mn Auger, respectively. The Mn $2 p$ spectrum shows 4 characteristic peaks, with the strongest components at $(641.9 \pm 0.1) \mathrm{eV}$ and $(653.5 \pm 0.1) \mathrm{eV}$ corresponding to the $2 \mathrm{p}_{3 / 2}$ and $2 \mathrm{p}_{1 / 2}$ peaks (with 2:1 area ratio) of $\mathrm{Mn}^{3+}$. Those at slightly higher binding energies of $(643.7 \pm 0.1) \mathrm{eV}$ and $(655.3 \pm 0.1) \mathrm{eV}$ can be associated with $\mathrm{Mn}$ in the oxidation state 4+. We note that the peak area ratio matches the ratio of $\mathrm{Mn}^{3+}$ to $\mathrm{Mn}^{4+}$ anticipated from the amount of strontium precursor used in the synthesis (see also Tab. S1 for a summary of the quantitative analysis). As expected, the $\mathrm{Sr} 3 \mathrm{~d}$ spectrum only shows 2 dominant peaks at $(133.2 \pm 0.1) \mathrm{eV}$ and $(134.9 \pm 0.1) \mathrm{eV}$ for the $3 d_{5 / 2}$ and $3 d_{3 / 2}$ levels.

As mentioned before, the film composition was also determined by ${ }^{4} \mathrm{He}^{+} \mathrm{RBS}$. Fig. $4 \mathbf{e}$ shows a representative spectrum and the results from curve fitting, the latter of which are in fair agreement with the $\mathrm{La} / \mathrm{Sr} / \mathrm{Mn}$ ratio from XPS quantitative analysis. Accordingly, the LSMO30 thin films can be described as $\mathrm{La}_{0.69} \mathrm{Sr}_{0.28} \mathrm{Mn}_{1.03} \mathrm{O}_{3 \pm \delta}$. Collectively, the data in Fig. 4, S2-S4 and Tab. S1 establish that the polymertemplated mesostructured LSMO films are chemically pure and the presence of second phases can be ruled out (within the limits of detection). In addition, they demonstrate the "complete" removal of carbon from the system, which emphasizes the good interconnectivity of the pores (i.e., there is no bottleneck to the mass transport). 

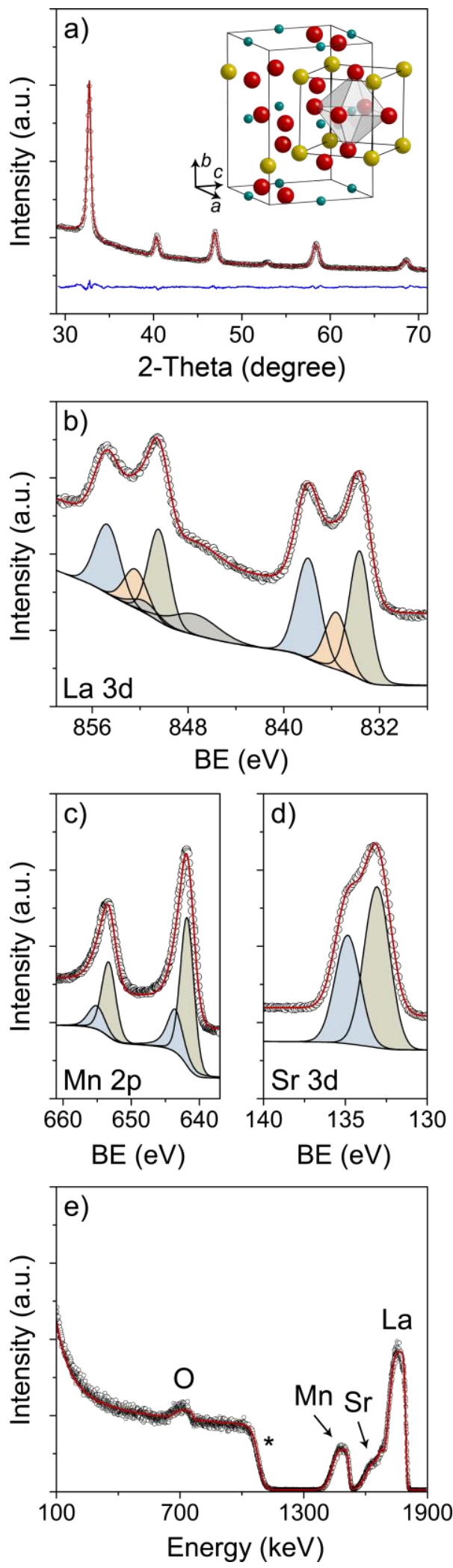

Figure 4. Crystallinity, chemical composition, and bonding configuration of polymertemplated mesostructured LSMO30 thin films. (a) Rietveld plot for ex situ GIWAXS data. The observed (black circles) and calculated patterns (red line) as well as the difference profile of the fit (blue line) are shown. The inset is a schematic of the orthorhombic unit-cell with oxygen in red, manganese in turquois, and 
lanthanum/strontium in yellow. XPS detailed measurements of the (b) La 3d, (c) Mn $2 p$, and (d) Sr 3d regions. The red lines are fits to the data assuming GaussianLorentzian peak shape. (e) RBS spectrum. The asterisk indicates the silicon edge at $1.13 \mathrm{MeV}$. The red line is a fit to the data.

The DC magnetic properties of the LSMO30 thin films were studied in plane by SQUID magnetometry. Experimental zero-field-cooled (ZFC) and field-cooled (FC) curves at 100 Oe are presented in Fig. 5a. The ZFC data show a relatively broad maximum centered at $290 \mathrm{~K}$, while the $\mathrm{FC}$ magnetization continues to increase with decreasing temperature and levels off at around $25 \mathrm{~K}$. Overall, the curves resemble the behavior expected from a superparamagnetic material. Fig. $\mathbf{5 b}$ shows fielddependent magnetization data for different temperatures. The saturation magnetization of virtually $3 \mu_{\mathrm{B}}$ per formula unit is reasonable. The coercive field $\left(H_{\mathrm{C}}\right)$ was found to decrease from $376 \mathrm{Oe}$ at $5 \mathrm{~K}$ to $43 \mathrm{Oe}$ at $300 \mathrm{~K}$ and to exhibit a square root dependence on $T^{1 / 2}$ (see inset of Fig. 5b). Such a dependence is usually observed for non-interacting, single-domain particles below the blocking temperature $\left(T_{\mathrm{B}}\right){ }^{51-53} T_{\mathrm{B}}$ was determined from a fit to the data to lie between 350 and $360 \mathrm{~K}$ (i.e., close to the magnetic transition temperature, $T_{\mathrm{C}}$ ).
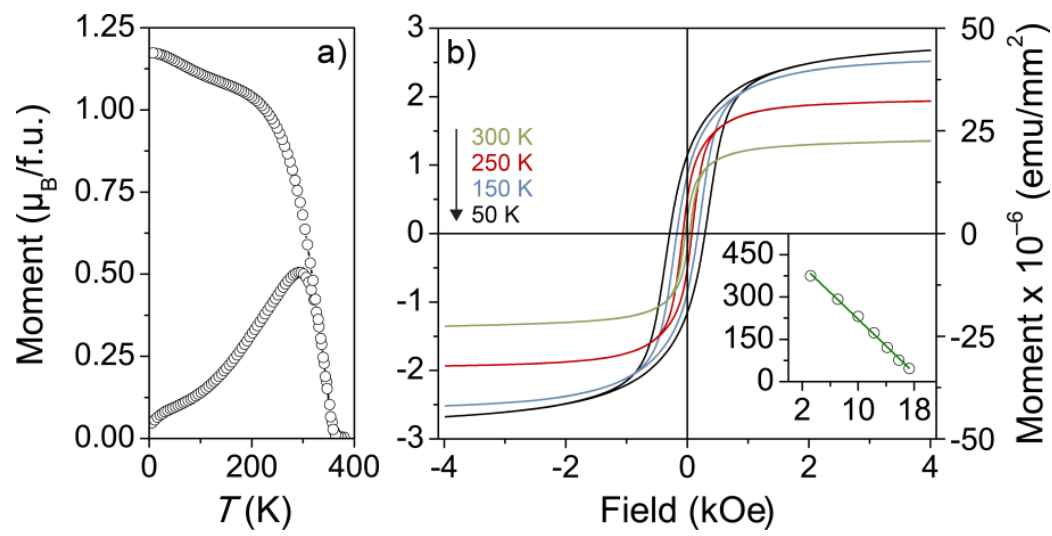

Figure 5. In plane DC magnetic properties of polymer-templated mesostructured LSMO30 thin films. (a) ZFC/FC curves at $100 \mathrm{Oe}$. (b) Field-dependent magnetization for different temperatures of $50 \mathrm{~K}, 150 \mathrm{~K}, 250 \mathrm{~K}$, and $300 \mathrm{~K}$. The inset shows the square root dependence of $H_{\mathrm{C}}(\mathrm{Oe})$ on $T^{1 / 2}\left(\mathrm{~K}^{1 / 2}\right)$, with $H_{\mathrm{C}}=H_{\mathrm{C} 0} \times\left[1-\left(T / T_{\mathrm{B}}\right)^{1 / 2}\right]$.

Because LSMO is a well-known colossal magnetoresistive material, the in plane magnetotransport properties of the polymer-templated mesostructured LSMO30 thin films were investigated as well. Both the negative low-field (LFMR) and high-field magnetoresistance (HFMR) was determined by measuring the electrical resistance $(R)$ as a function of temperature and magnetic field. The negative MR, following Eq. 1, is:

$$
\mathrm{MR}(\%)=[(R(\mathrm{H})-R(0)) / R(0)] \times 100
$$


Fig. 6a shows the temperature-dependent negative HFMR for different magnetic fields along with the measured electrical resistance with and without applied field. The electrical resistance always increases with decreasing temperature until a maximum is reached at around $270 \mathrm{~K}$. This maximum can be associated with the insulator-to-metal transition $\left(T_{\mathrm{IM}}\right)$. Below $T_{\mathrm{IM}}$, the electrical resistance decreases until $T \approx 54 \mathrm{~K}$ and $40 \mathrm{~K}$ at $H=0 \mathrm{Oe}$ and $70 \mathrm{kOe}$, respectively, and then increases again due to electron-electron interactions and/or spin-disorder scattering. This behavior is consistent with literature observations. ${ }^{54-56}$ In contrast, the negative HFMR steadily increases with decreasing temperature to nearly $45 \%$ at $T=5 \mathrm{~K}$. This behavior is different from epitaxial layers, but has been reported before for (polycrystalline) LSMO nanomaterials. Also, as is evident, the MR effect increases with increasing field, making the mesoporous thin films promising for application in magnetic field sensors that can operate over a wide range of temperatures.

The negative LFMR in the range of $\pm 4 \mathrm{kOe}$ is shown for different temperatures in Fig. 6b-e. In agreement with theory, the films exhibit the highest electrical resistance, and therefore the lowest absolute MR value, around $H_{\mathrm{C}}$. The primary reason is the low relative orientation of spin-polarized carriers at the Fermi energy; "strong" electron scattering at domain walls results in high electrical resistance. When $H>H_{\mathrm{C}}$, the electrical resistance decreases significantly due to facilitated electron transport across the grain boundaries and the MR effect increases. As can be seen from Fig. 6b-e, the absolute LFMR decreases with increasing temperature $(\approx 25 \%$ at $5 \mathrm{~K}, 17 \%$ at $100 \mathrm{~K}, 8 \%$ at $200 \mathrm{~K}$, and $2 \%$ at $300 \mathrm{~K})$. These values are similar or slightly higher than those obtained from solution-processed LSMO or related perovskite-type manganese oxides. ${ }^{57}$ 

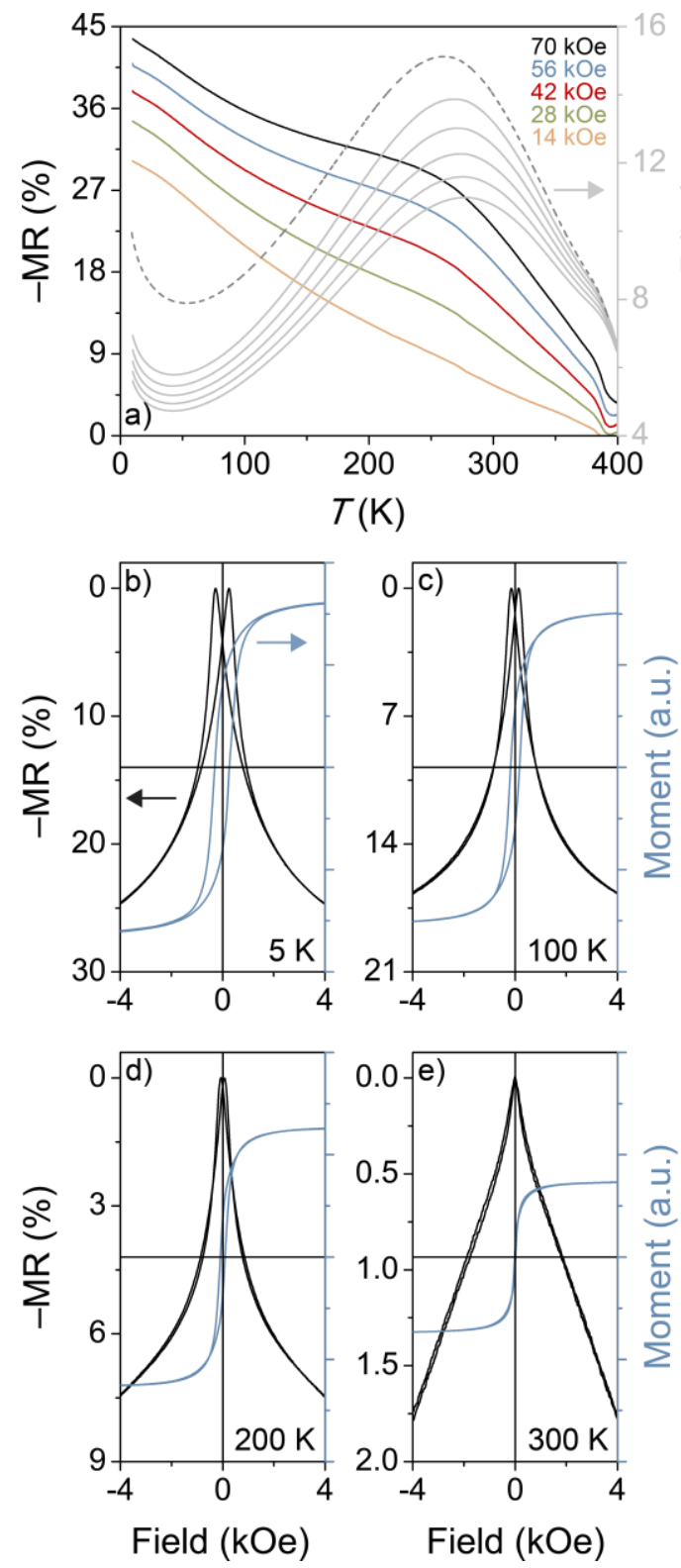

Figure 6. Magnetotransport properties of polymer-templated mesostructured LSMO30 thin films. (a) Temperature-dependent electrical resistance and the corresponding negative HFMR effect for different magnetic fields applied parallel to the film plane. The electrical resistance measured without field is shown as dashed line. (b-e) Magnetic hysteresis loops (blue) at $5 \mathrm{~K}, 100 \mathrm{~K}, 200 \mathrm{~K}$, and $300 \mathrm{~K}$ along with the negative LFMR effect (black).

Finally, we demonstrate that carriers can be electrostatically induced into the films via capacitive double-layer charging. This is intriguing as it allows tuning of the magnetization in situ. To this end, gating experiments were performed inside a SQUID magnetometer using two-electrode cells, with high-surface-area carbon fiber cloth and mesoporous LSMO30 serving as counter and working electrodes, respectively. For reasons of feasibility, an ionic liquid, namely diethylmethyl(2- 
methoxyethyl)ammonium bis(trifluoromethylsulfonyl)imide, was used as the electrolyte. We note that this particular ionic liquid is characterized by a large temperature window in which it remains in the liquid state (see Fig. 7a). Assuming that electrostatic carrier doping is equivalent to chemical doping, non-Faradaic energy storage should, in principle, be accompanied by both a shift in $T_{\mathrm{C}}$ (at "high" temperatures) and a change in total magnetization (at "low" temperatures). More specifically, positive $\left(\mathrm{h}^{+}\right)$and negative $\left(\mathrm{e}^{-}\right)$charge accumulation should result in an increase and decrease in $T_{\mathrm{C}}$ - due to changes in exchange interactions between the manganese ions $\left(\mathrm{Mn}^{3+} / \mathrm{Mn}^{3+}, \mathrm{Mn}^{4+} / \mathrm{Mn}^{3+}\right.$, and $\left.\mathrm{Mn}^{4+} / \mathrm{Mn}^{4+}\right)$ - and in a decrease and increase in total magnetic moment, respectively. The latter can be understood in terms of a simple band-filling model as each accumulated electron contributes an additional $\mu_{\mathrm{B}}$ to the total moment. To prove these hypotheses, temperaturedependent measurements were performed on the LSMO30 thin films. Here the potential was applied for a period of $60 \mathrm{~min}$ under isothermal conditions, and then the temperature was ramped to $5 \mathrm{~K}$. The magnetic field was set to 500 Oe during the experiment. As is seen in Fig. $7 \mathrm{a}, \mathrm{h}^{+}$accumulation indeed led to a shift in $T_{\mathrm{C}}$ to higher temperatures (relative to the negative charge accumulation state), and $\mathrm{e}^{-}$ accumulation to a higher saturation magnetization at low temperatures. Thus, these data establish that in situ manipulation of the magnetic phase diagram of LSMO is possible. They also indicate that the most significant effect can be expected around the magnetic transition temperature. To gain more insight, cyclic voltammetric (CV) experiments were conducted under isothermal conditions. Notably, both the magnetization changes and surface current density, or surface charge density (amount of charge per unit surface area), were monitored simultaneously. Fig. 7b presents CV curves obtained at $330 \mathrm{~K}$ in the potential range from $-600 \mathrm{mV}$ to +750 $\mathrm{mV}$. They barely changed with cycling, thereby already indicating good reversibility; the quasi rectangular shape is characteristic of supercapacitors or ultracapacitors. ${ }^{58-}$ ${ }^{60} \mathrm{In} \mathrm{Fig.} \mathrm{7d,} \mathrm{the} \mathrm{corresponding} \mathrm{relative} \mathrm{change} \mathrm{in} \mathrm{magnetization} \mathrm{at} 500 \mathrm{Oe}$ and the surface charge density both as a function of time are shown. The curves follow the very same shape, thus corroborating that the tuning effect is, in fact, reversible in nature. The peak-to-peak magnetization modulation was equal to $6.0 \%$, which is among the largest values reported for ionic liquid-gated mixed-valence manganese oxides. $^{10,12}$

To provide further evidence that the tuning, which is "positive" at high temperatures and "negative" at low temperatures (with respect to $\mathrm{h}^{+}$accumulation), is dependent upon the surface charge density at isothermal conditions, gating experiments were performed at the lowest temperature at which the electrolyte still exists in the liquid state. Fortunately, this temperature was in the intermediate range. In other words, it was neither too high, so that the effect on $T_{\mathrm{C}}$ is the major mechanism, nor too low, so that the tuning is primarily determined by the above mentioned band-filling model. In these experiments, the applied field was set to $1000 \mathrm{Oe}$ (to reach magnetic saturation). Fig. 7c shows CV curves at $220 \mathrm{~K}$ from $-600 \mathrm{mV}$ to $+450 \mathrm{mV}$ and -1800 $\mathrm{mV}$ to $+1500 \mathrm{mV}$. Again, their shape is characteristic of double-layer charging. The 
reason for the presence of subtle peaks around the cut-off potentials (see green curve in Fig. 7c) is not well understood, but might be related to electrolyte decomposition reactions or pseudocapacitive behavior. However, the fact that the CV curves are nearly perfectly superimposed indicates that the charge storage process is highly reversible. The magnetic response is shown in Fig. 7e,f along with the surface charge density versus the time. As expected, for very small perturbations (i.e., low surface charge), the curves are not in phase, but rather are anti-phase (see Fig. 7e). We believe that this is due to the fact that positive charging is accompanied by a decrease in magnetic moment by approx. $1 \mu_{\mathrm{B}} / \mathrm{h}^{+}$, while negative charging leads to the opposite effect. However, when the surface charge density is increased, the shift in $T_{\mathrm{C}}$ becomes noticeable such that suddenly both tuning mechanisms are present simultaneously. This can be seen from the data in Fig. 7f; now, the magnetic response curve clearly exhibits an out-of-phase (low charge density) and an inphase (high charge density) component. Overall, the experiments demonstrate clearly the "competing" nature of both mechanisms in perovskite-type mixed-valence manganese oxides. On the one hand, the magnetic exchange interactions are strengthened or weakened by inducing carriers into the material and, on the other hand, the saturation at low temperatures is altered, following a simple band-filling model. 

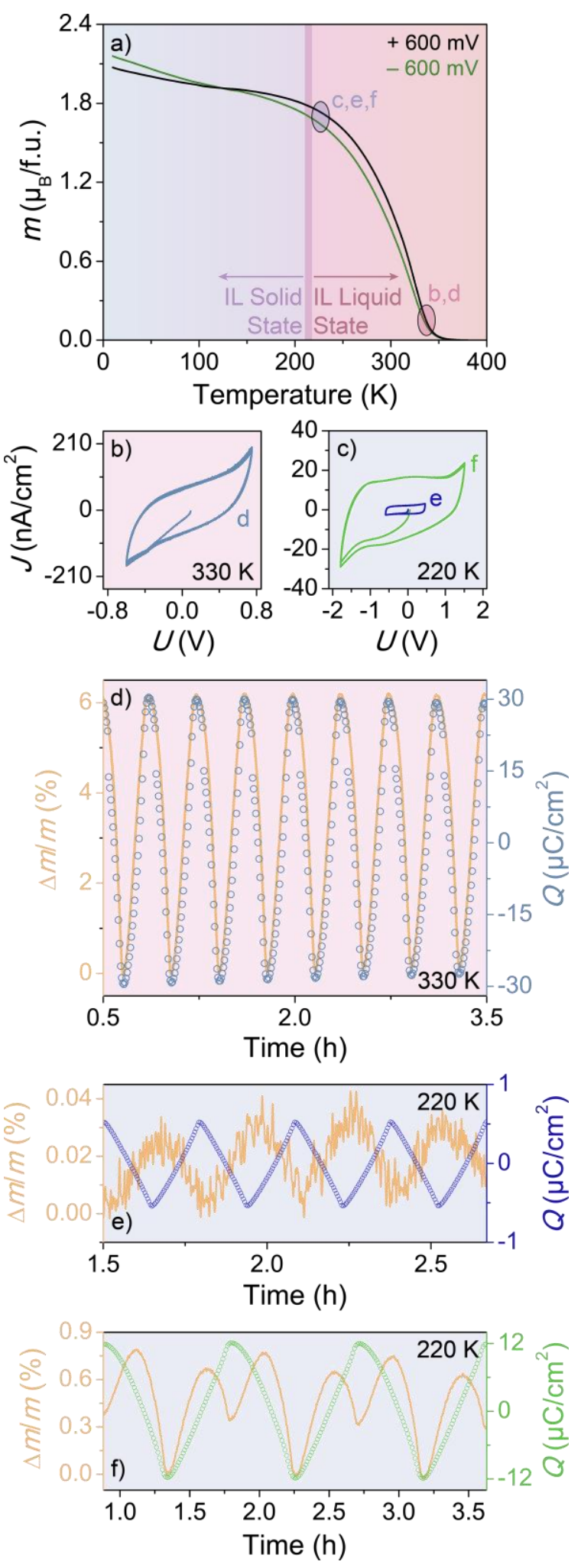

Figure 7. Magnetic tuning of polymer-templated mesostructured LSMO30 thin films. (a) FC curves at $500 \mathrm{Oe}$ for different charging states. (b, c) Voltammetry sweeps at $330 \mathrm{~K}$ from $-600 \mathrm{mV}$ to $+750 \mathrm{mV}$ ( 8 cycles) and at $220 \mathrm{~K}$ from $-600 \mathrm{mV}$ to $+450 \mathrm{mV}$ (4 cycles) and $-1800 \mathrm{mV}$ to $+1500 \mathrm{mV}$ ( 3 cycles). The scan rate was $2 \mathrm{mV} / \mathrm{s}$. (d-f) Relative changes in magnetization at $500 \mathrm{Oe}(\mathrm{d})$ and at $1000 \mathrm{Oe}(\mathrm{e}, \mathrm{f})$ along with the surface charge density versus the time. The surface charge density was calculated 
by measuring the current and voltage and knowing the total surface area of the films (from STEM tomography).

\title{
Conclusion
}

Thermally stable, 3D cubic mesoporous $\mathrm{La}_{1-x} \mathrm{Sr}_{x} \mathrm{MnO}_{3}$ (LSMO) was prepared as a thin film by evaporation-induced self-assembly. The sol-gel-derived material with varying Sr contents from $x \approx 0.15$ to 0.30 was shown by various techniques to be single phase and well-defined in terms of composition and pore structure after full crystallization at $700^{\circ} \mathrm{C}$. The Sr-rich LSMO phase exhibited ferromagnetic behavior below the blocking temperature of around $350 \mathrm{~K}$, with enhanced coercivity in comparison with bulk material due to the unique combination of nanocrystallinity and mesoporosity. Magnetotransport measurements demonstrated that the polymertemplated films behave differently from epitaxially grown LSMO, which was particularly evident from the large low-field magnetoresistive effect. However, we believe that both the high- and low-field magnetoresistance can be increased further by affecting the intrinsic domain structure and charge transport pathways (e.g., by coating the pore walls with a thin layer of a different material using atomic layer deposition). Furthermore, the manipulation of magnetic properties or, more specifically, of total magnetization and Curie temperature was achieved in situ over a broad temperature range by non-Faradaic capacitive charging. Overall, the results suggest that electrostatic hole and electron doping via ionic liquid gating in highsurface-area materials offers the intriguing possibility of strongly tuning the magnetic phase diagram to explore new functionality.

\section{Associated Content}

Supporting Information

Electron microscopy images, GIWAXS patterns, and XPS survey spectra of mesoporous LSMO15, LSMO225, and LSMO30 thin films; and RBS spectra and XPS detailed measurements of the La $3 d, M n 2 p$, and Sr $3 d$ regions for mesoporous LSMO15 and LSMO225 thin films.

\author{
Author Information \\ *Phone: +49 721 60828923, E-mail: christian.reitz@kit.edu \\ *Phone: +49 721 60828827, E-mail: torsten.brezesinski@kit.edu
}




\section{Notes}

The authors declare no competing financial interest.

\section{Acknowledgements}

We thank Robert Kruk and Philipp M. Leufke for fruitful discussions, and Christian Grupe for graphical assistance. Parts of this research were carried out at the light source PETRA III at DESY, a member of the Helmholtz Association (HGF). We thank Matthias Schwartzkopf for assistance in using beamline P03. This project was supported by the Center of Materials Research (LaMa) at Justus-Liebig-University Giessen. Financial support by the German Academic Exchange Service (T.B.) and the German Research Foundation (H.H., grant no. DA 1781/1-1 and HA 1344/34-1) is gratefully acknowledged.

\section{References}

(1) Dagotto, E. Nanoscale Phase Separation and Colossal Magnetoresistance; Dagotto, E., Springer: Berlin, 2003; Vol. 136.

(2) Jiang, S. P.; Love, J. G. Observation of Structural Change Induced by Cathodic Polarization on (La,Sr) $\mathrm{MnO}_{3}$ Electrodes of Solid Oxide Fuel Cells. Solid State Ionics 2003, 158 (1-2), 45-53.

(3) De Souza, R. A.; Islam, M. S.; Ivers-Tiffée, E. Formation and Migration of Cation Defects in the Perovskite Oxide $\mathrm{LaMnO}_{3}$. J. Mater. Chem. 1999, 9 (7), $1621-1627$

(4) Gor'kov, L. P.; Kresin, V. Z. Mixed-Valence Manganites: Fundamentals and Main Properties. Phys. Rep. 2004, 400 (3), 149-208.

(5) Coey, J. M. D.; Viret, M.; von Molnár, S. Mixed-Valence Manganites. Adv. Phys. 1999, 48 (2), 167-293.

(6) Bason, Y.; Klein, L.; Yau, J. B.; Hong, X.; Ahn, C. H. Giant Planar Hall Effect in Colossal Magnetoresistive $\mathrm{La}_{0.84} \mathrm{Sr}_{0.16} \mathrm{MnO}_{3}$ Thin Films. Appl. Phys. Lett. 2004, 84 (14), 2593-2595.

(7) Viret, M.; Drouet, M.; Nassar, J.; Contour, J. P.; Fermon, C.; Fert, A. Low-Field Colossal Magnetoresistance in Manganite Tunnel Spin Valves. Europhys. Lett. 1997, 39 (5), 545-550.

(8) Haghiri-Gosnet, A.-M.; Renard, J.-P. CMR Manganites: Physics, Thin Films and Devices. J. Phys. D. Appl. Phys. 2003, 36 (8), R127-R150.

(9) Dagotto, E.; Hotta, T.; Moreo, A. Colossal Magnetoresistant Materials: The Key Role of Phase Separation. Phys. Rep. 2001, 344 (1-3), 1-153. 
(10) Reitz, C.; Leufke, P. M.; Schneider, R.; Hahn, H.; Brezesinski, T. Large Magnetoresistance and Electrostatic Control of Magnetism in Ordered Mesoporous $\mathrm{La}_{1-x} \mathrm{Ca}_{x} \mathrm{MnO}_{3}$ Thin Films. Chem. Mater. 2014, 26 (19), 57455751.

(11) Leufke, P. M.; Kruk, R.; Brand, R. A.; Hahn, H. In Situ Magnetometry Studies of Magnetoelectric LSMO/PZT Heterostructures. Phys. Rev. B 2013, 87 (9), 94416.

(12) Mishra, A. K.; Darbandi, A. J.; Leufke, P. M.; Kruk, R.; Hahn, H. Room Temperature Reversible Tuning of Magnetism of Electrolyte-Gated $\mathrm{La}_{0.75} \mathrm{Sr}_{0.25} \mathrm{MnO}_{3}$ Nanoparticles. J. Appl. Phys. 2013, 113 (3), 33913.

(13) Molegraaf, H. J. A.; Hoffman, J.; Vaz, C. A. F.; Gariglio, S.; Van Der Morel, D.; Ahn, C. H.; Triscone, J. M. Magnetoelectric Effects in Complex Oxides with Competing Ground States. Adv. Mater. 2009, 21 (34), 1-5.

(14) Lu, H.; George, T. A.; Wang, Y.; Ketsman, I.; Burton, J. D.; Bark, C. W.; Ryu, S.; Kim, D. J.; Wang, J.; Binek, C.; Dowben, P. A.; Sokolov, A.; Eom, C. B.; Tsymbal, E. Y.; Gruverman, A. Electric Modulation of Magnetization at the $\mathrm{BaTiO}_{3} / \mathrm{La}_{0.67} \mathrm{Sr}_{0.33} \mathrm{MnO}_{3}$ Interfaces. Appl. Phys. Lett. 2012, 100 (23), 232904.

(15) Vaz, C. A. F.; Hoffman, J.; Segal, Y.; Reiner, J. W.; Grober, R. D.; Zhang, Z.; Ahn, C. H.; Walker, F. J. Origin of the Magnetoelectric Coupling Effect in $\mathrm{Pb}\left(\mathrm{Zr}_{0.2} \mathrm{~T}_{0.8}\right) \mathrm{O}_{3} / \mathrm{La}_{0.8} \mathrm{Sr}_{0.2} \mathrm{MnO}_{3}$ Multiferroic Heterostructures. Phys. Rev. Lett. 2010, 104 (12), 127202.

(16) Brivio, S.; Cantoni, M.; Petti, D.; Bertacco, R. Near-Room-Temperature Control of Magnetization in Field Effect Devices Based on $\mathrm{La}_{0.67} \mathrm{Sr}_{0.33} \mathrm{MnO}_{3}$ Thin Films. J. Appl. Phys. 2010, 108 (11), 113906.

(17) Reitz, C.; Suchomski, C.; Wang, D.; Hahn, H.; Brezesinski, T. In Situ Tuning of Magnetization via Topotactic Lithium Insertion in Ordered Mesoporous Lithium Ferrite Thin Films. J. Mater. Chem. C 2016, 4 (38), 8889-8896.

(18) Dasgupta, S.; Das, B.; Knapp, M.; Brand, R. A.; Ehrenberg, H.; Kruk, R.; Hahn, $\mathrm{H}$. Intercalation-Driven Reversible Control of Magnetism in Bulk Ferromagnets. Adv. Mater. 2014, 26 (27), 4639-4644.

(19) Wei, G.; Wei, L.; Wang, D.; Tian, Y.; Chen, Y.; Yan, S.; Mei, L.; Jiao, J. Reversible Control of the Magnetization of $\mathrm{Fe}_{3} \mathrm{O}_{4}$ via Lithium Ions. RSC Adv. 2017, 7 (5), 2644-2649.

(20) Weissmuller, J. Charge-Induced Reversible Strain in a Metal. Science 2003, 300 (5617), 312-315.

(21) Takamura, Y.; Chopdekar, R. V; Arenholz, E.; Suzuki, Y. Control of the Magnetic and Magnetotransport Properties of $\mathrm{La}_{0.67} \mathrm{Sr}_{0.33} \mathrm{MnO}_{3}$ Thin Films through Epitaxial Strain. Appl. Phys. Lett. 2008, 92 (16), 162504.

(22) Zou, C.; Chen, Y.-F.; Li, P.-J.; Fan, R.; Peng, B.; Zhang, W.-X.; Wang, Z.-G.; Hao, X.; Liu, J.-B.; Zhang, W.-L.; Li, Y.-R.; Li, R.-W. Structure and Properties of Epitaxial Perovskite $\mathrm{Pb}\left(\mathrm{Zr}_{0.52} \mathrm{Ti}_{0.48}\right) \mathrm{O}_{3} / \mathrm{La}_{0.7} \mathrm{Sr}_{0.3} \mathrm{MnO}_{3}$ Heterostructures. J. 
Appl. Phys. 2012, 111 (7), 07D718.

(23) Chen, Y.-H.; $\mathrm{Wu}, \mathrm{T} .-\mathrm{B}$. Strain Effects on the Ferromagnetic and Electronic Properties in $\left(\mathrm{La}_{0.91}, \mathrm{Sr}_{0.09}\right) \mathrm{MnO}_{3}$ Thin Films. Electrochem. Solid-State Lett. 2007, 10 (5), G29.

(24) Spaldin, N. A.; Fiebig, M. Materials Science. The Renaissance of Magnetoelectric Multiferroics. Science 2005, 309 (5733), 391-392.

(25) Eerenstein, W.; Wiora, M.; Prieto, J. L.; Scott, J. F.; Mathur, N. D. Giant Sharp and Persistent Converse Magnetoelectric Effects in Multiferroic Epitaxial Heterostructures. Nat. Mater. 2007, 6 (5), 348-351.

(26) Brinker, C. J.; Lu, Y.; Sellinger, A.; Fan, H. Evaporation-Induced SelfAssembly: Nanostructures Made Easy. Adv. Mater. 1999, 11 (7), 579-585.

(27) Grosso, D.; Cagnol, F.; Soler-Illia, G. J. de A. A.; Crepaldi, E. L.; Amenitsch, H.; Brunet-Bruneau, A.; Bourgeois, A.; Sanchez, C. Fundamentals of Mesostructuring Through Evaporation-Induced Self-Assembly. Adv. Funct. Mater. 2004, 14 (4), 309-322.

(28) Lu, Y.; Ganguli, R.; Drewien, C. A.; Anderson, M. T.; Brinker, C. J.; Gong, W.; Guo, Y.; Soyez, H.; Dunn, B.; Huang, M. H.; Zink, J. I. Continuous Formation of Supported Cubic and Hexagonal Mesoporous Films by Sol-Gel Dip-Coating. Nature 1997, 389 (6649), 364-368.

(29) Rawolle, M.; Niedermeier, M. A.; Kaune, G.; Perlich, J.; Lellig, P.; Memesa, M.; Cheng, Y.-J.; Gutmann, J. S.; Müller-Buschbaum, P. Fabrication and Characterization of Nanostructured Titania Films with Integrated Function from Inorganic-Organic Hybrid Materials. Chem. Soc. Rev. 2012, 41 (15), 51315142.

(30) Innocenzi, P.; Malfatti, L. Mesoporous Thin Films: Properties and Applications. Chem. Soc. Rev. 2013, 42 (9), 4198-4216.

(31) Sanchez, C.; Boissière, C.; Grosso, D.; Laberty, C.; Nicole, L. Design, Synthesis, and Properties of Inorganic and Hybrid Thin Films Having Periodically Organized Nanoporosity. Chem. Mater. 2008, 20 (3), 682-737.

(32) Deng, Y.; Wei, J.; Sun, Z.; Zhao, D. Large-Pore Ordered Mesoporous Materials Templated from Non-Pluronic Amphiphilic Block Copolymers. Chem. Soc. Rev. 2013, 42 (9), 4054-4070.

(33) Gu, D.; Schüth, F. Synthesis of Non-Siliceous Mesoporous Oxides. Chem. Soc. Rev. 2013, 43 (1), 313-344.

(34) Ren, Y.; Ma, Z.; Bruce, P. G. Ordered Mesoporous Metal Oxides: Synthesis and Applications. Chem. Soc. Rev. 2012, 41 (14), 4909-4927.

(35) Petkovich, N. D.; Stein, A. Controlling Macro- and Mesostructures with Hierarchical Porosity through Combined Hard and Soft Templating. Chem. Soc. Rev. 2013, 42 (9), 3721-3739.

(36) Choi, S. Y.; Mamak, M.; Coombs, N.; Chopra, N.; Ozin, G. A. Thermally Stable 
Two-Dimensional Hexagonal Mesoporous Nanocrystalline Anatase, Meso-NcTiO2: Bulk and Crack-Free Thin Film Morphologies. Adv. Funct. Mater. 2004, 14 (4), 335-344.

(37) Smarsly, B.; Grosso, D.; Brezesinski, T.; Pinna, N.; Boissière, C.; Antonietti, M.; Sanchez, C. Highly Crystalline Cubic Mesoporous $\mathrm{TiO}_{2}$ with 10-nm Pore Diameter Made with a New Block Copolymer Template. Chem. Mater. 2004, 16 (15), 2948-2952.

(38) Yue, J.; Suchomski, C.; Brezesinski, T.; Smarsly, B. M. Polymer-Templated Mesoporous $\mathrm{Li}_{4} \mathrm{Ti}_{5} \mathrm{O}_{12}$ as a High-Rate and Long-Life Anode Material for Rechargeable Li-Ion Batteries. ChemNanoMat 2015, 1 (6), 415-421.

(39) Elm, M. T.; Hofmann, J. D.; Suchomski, C.; Janek, J.; Brezesinski, T. Ionic Conductivity of Mesostructured Yttria-Stabilized Zirconia Thin Films with Cubic Pore Symmetry-On the Influence of Water on the Surface Oxygen Ion Transport. ACS Appl. Mater. Interfaces 2015, 7 (22), 11792-11801.

(40) Weidmann, C.; Brezesinski, K.; Suchomski, C.; Tropp, K.; Grosser, N.; Haetge, J.; Smarsly, B. M.; Brezesinski, T. Morphology-Controlled Synthesis of Nanocrystalline $\eta-\mathrm{Al}_{2} \mathrm{O}_{3}$ Thin Films, Powders, Microbeads, and Nanofibers with Tunable Pore Sizes from Preformed Oligomeric Oxo-Hydroxo Building Blocks. Chem. Mater. 2012, 24 (3), 486-494.

(41) Kremer, J. R.; Mastronarde, D. N.; McIntosh, J. R. Computer Visualization of Three-Dimensional Image Data Using IMOD. J. Struct. Biol. 1996, 116 (1), 7176.

(42) Bruns, S.; Hara, T.; Smarsly, B. M.; Tallarek, U. Morphological Analysis of Physically Reconstructed Capillary Hybrid Silica Monoliths and Correlation with Separation Efficiency. J. Chromatogr. A 2011, 1218 (31), 5187-5194.

(43) Hormann, K.; Müllner, T.; Bruns, S.; Höltzel, A.; Tallarek, U. Morphology and Separation Efficiency of a New Generation of Analytical Silica Monoliths. J. Chromatogr. A 2012, 1222, 46-58.

(44) Benecke, G.; Wagermaier, W.; Li, C.; Schwartzkopf, M.; Flucke, G.; Hoerth, R.; Zizak, I.; Burghammer, M.; Metwalli, E.; Müller-Buschbaum, P.; Trebbin, M.; Förster, S.; Paris, O.; Roth, S. V; Fratzl, P. A Customizable Software for Fast Reduction and Analysis of Large X-Ray Scattering Data Sets: Applications of the New DPDAK Package to Small-Angle X-Ray Scattering and GrazingIncidence Small-Angle X-Ray Scattering. J. Appl. Crystallogr. 2014, 47 (Pt 5), 1797-1803.

(45) Mayer, M. SIMNRA, a Simulation Program for the Analysis of NRA, RBS and ERDA. In AIP Conference Proceedings; AIP, 1999; Vol. 475, pp 541-544.

(46) Friedman, S. P.; Seaton, N. A. A Corrected Tortuosity Factor for the Network Calculation of Diffusion Coefficients. Chem. Eng. Sci. 1995, 50 (5), 897-900.

(47) Stoeckel, D.; Kübel, C.; Loeh, M. O.; Smarsly, B. M.; Tallarek, U. Morphological Analysis of Physically Reconstructed Silica Monoliths with Submicrometer Macropores: Effect of Decreasing Domain Size on Structural 
Homogeneity. Langmuir 2015, 31 (26), 7391-7400.

(48) Stoeckel, D.; Kübel, C.; Hormann, K.; Höltzel, A.; Smarsly, B. M.; Tallarek, U. Morphological Analysis of Disordered Macroporous-Mesoporous Solids Based on Physical Reconstruction by Nanoscale Tomography. Langmuir 2014, 30 (30), 9022-9027.

(49) Suchomski, C.; Reitz, C.; Pajic, D.; Jaglicic, Z.; Djerdj, I.; Brezesinski, T. Large-Pore Mesoporous $\mathrm{Ho}_{3} \mathrm{Fe}_{5} \mathrm{O}_{12}$ Thin Films with a Strong RoomTemperature Perpendicular Magnetic Anisotropy by Sol-Gel Processing. Chem. Mater. 2014, 26 (7), 2337-2343.

(50) Reitz, C.; Haetge, J.; Suchomski, C.; Brezesinski, T. Facile and General Synthesis of Thermally Stable Ordered Mesoporous Rare-Earth Oxide Ceramic Thin Films with Uniform Mid-Size to Large-Size Pores and Strong Crystalline Texture. Chem. Mater. 2013, 25 (22), 4633-4642.

(51) Stoner, E. C.; Wohlfarth, E. P. A Mechanism of Magnetic Hysteresis in Heterogeneous Alloys. Philos. T. R. Soc. A. 1948, 240 (826), 599-642.

(52) Nunes, W. C.; Folly, W. S. D.; Sinnecker, J. P.; Novak, M. A. Temperature Dependence of the Coercive Field in Single-Domain Particle Systems. Phys. Rev. B 2004, 70 (1), 14419.

(53) Bean, C. P.; Livingston, J. D. Superparamagnetism. J. Appl. Phys. 1959, 30 (4), S120.

(54) Dey, P.; Nath, T. Effect of Grain Size Modulation on the Magneto- and Electronic-Transport Properties of $\mathrm{La}_{0.7} \mathrm{Ca}_{0.3} \mathrm{MnO}_{3}$ Nanoparticles: The Role of Spin-Polarized Tunneling at the Enhanced Grain Surface. Phys. Rev. B 2006, 73 (21), 214425.

(55) Balcells, L.; Fontcuberta, J.; Martínez, B.; Obradors, X. High-Field Magnetoresistance at Interfaces in Manganese Perovskites. Phys. Rev. B 1998, 58 (22), 14697-14700.

(56) Li, P. G.; Lei, M.; Yang, H.; Tang, H. L.; Guo, Y. F.; Tang, W. H. Magnetic and Electrical Transport Properties of Nanostructured $\mathrm{La}_{0.67} \mathrm{Ca}_{0.33} \mathrm{MnO}_{3}$ Networks. J. Alloys Compd. 2008, 460 (1-2), 60-63.

(57) Vengalis, B.; Grigaliūnaitè-Vonsevičienè, G.; Maneikis, A.; Klimantavičius, J.; Juškėnas, R.; Mažeika, K. Low-Field Magnetoresistance and Switching Behavior of Polycrystalline $\mathrm{La}_{0.66} \mathrm{Sr}_{0.34} \mathrm{MnO}_{3} / \mathrm{YSZ}(001)$ Films with Columnar Grain Structure. Thin Solid Films 2017, 625, 42-48.

(58) Burke, A. Ultracapacitors: Why, How, and Where Is the Technology. J. Power Sources 2000, 91 (1), 37-50.

(59) Kotz, R.; Carlen, M.; Kötz, R.; Kötz, R.; Carlen, M.; Carlen, M. Principles and Applications of Electrochemical Capacitors. Electrochim. Acta 2000, 45 (1516), 2483-2498.

(60) Stoller, M. D.; Park, S.; Yanwu, Z.; An, J.; Ruoff, R. S. Graphene-Based Ultracapacitors. Nano Lett. 2008, 8 (10), 3498-3502. 
TOC Graphics

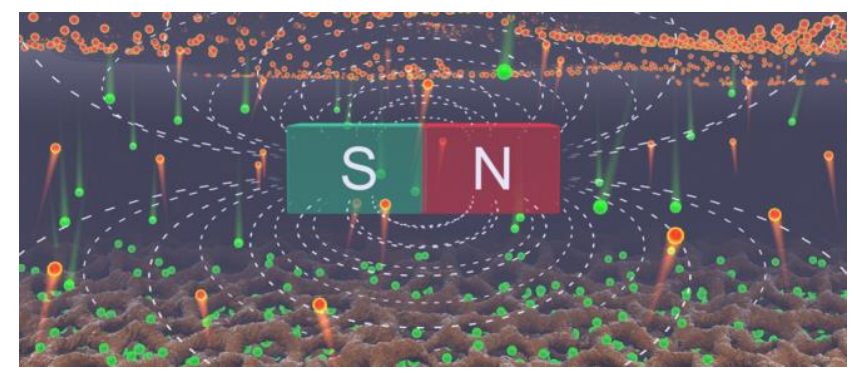

